\title{
Coordinating Centers and Multi-Practice-based Research Network (PBRN) Research
}

\author{
James W. Mold, MD, MPH, Paula Darby Lipman, PhD, and Stephen J. Durako, BA
}

Practice-based research networks (PBRNs) have emerged as laboratories in which to address important primary care challenges. In 2011, the Agency for Healthcare Research and Quality's PBRN database included more than 130 networks, most regional and some national, with member practices in every state. Regional networks may have certain advantages over national networks with respect to practice recruitment and project quality control because of closer relationships and shorter distances. However, national networks often can achieve larger numbers of practices with greater diversity, resulting in broader generalizability of results. Increasingly, regional networks are collaborating on multinetwork projects, but this creates significant study coordination challenges. A potential solution is to incorporate PBRN coordinating centers similar to those used in many National Institutes of Health and industrysponsored multi-center clinical trials. In this article, we discuss the potential functions of a coordinating center in multi-region PBRN studies based on our experience with 2 recent studies. ( $\mathrm{J}$ Am Board Fam Med 2012;25:577-581.)

Keywords: Coordinating Center, Practice-based Research, Practice-based Research Networks, Primary Health Care

\section{The Evolution of Practice-based Research Networks}

The history of practice-based research networks (PBRNs) has been summarized by Green and Hickner ${ }^{1}$ in an earlier issue of this fournal. Many of the early primary care networks evolved from within the specialty of Family Medicine. Their purpose was to learn more about the diagnosis and management of common medical conditions seen in primary care. Now, 4 decades later, the Agency for Health care Research and Quality (AHRQ) has registered more than 130 primary care PBRNs in the United States, some national in scope (20\%), but most regional, involving single cities $(22 \%)$,

This article was externally peer reviewed.

Submitted 28 October 2011; revised 27 February 2012; accepted 14 March 2012.

From the Department of Family and Preventive Medicine, University of Oklahoma Health Sciences Center, Oklahoma City (JWM); and Westat, Rockville, MD (PDL, SJD).

Funding: none.

Conflict of interest: PDL and SJD are employees of Westat, a private, for-profit research organization that provides coordinating center functions. JWM has contracted with Westat for coordinating center functions.

Corresponding author: James W. Mold, MD, MPH, University of Oklahoma Health Sciences Center, Department of Family and Preventive Medicine, 900 NE 10th Street, Oklahoma City, OK 73104 (E-mail: james-mold@ouhsc.edu). states $(28 \%)$, or regions of the country $(30 \%)$. Their research agendas include theoretical and methodological research, research focused on the function of primary care itself (process of care), clinical research, and health systems research. ${ }^{2}$ Many have evolved into learning communities that attract, develop, and share resources and support continuous quality improvement efforts. $^{3}$

Each network type has both advantages and disadvantages. Local and regional networks often maintain close relationships with their members, which facilitates study recruitment and retention. Because of shorter travel distances, they are able to achieve tighter oversight during interventions and data collection and have a more visible presence within the community of practitioners. They also are more likely to be aware of the needs and interests of their members, to more effectively increase the readiness and capacity of practice sites to participate in research, and to build viable learning communities for dissemination of knowledge. However, smaller numbers of practices available to participate can limit the types of study designs in which regional PBRNs can participate and may impact the generalizability of research find- 
ings to other regions of the country or to other practice types or patient populations.

A major advantage of national and international networks is their ability to recruit larger numbers of practices of different types and sizes from more diverse settings, resulting in greater generalizability of results and, because of that, a competitive advantage when it comes to funding. However, these gains are potentially offset by a lack of a presence in the community, fewer opportunities for building relationships, and greater geographic distance between member practices.

For these reasons, and because regional PBRNs are interested in expanding the scope of research projects, several PBRN researchers have proposed that studies involving multiple regional networks may be the best way to achieve both quality control and generalizability. However, this calls for a mechanism to manage and coordinate the research processes across networks to ensure consistency and fidelity of recruitment and enrollment, to monitor intervention implementation, and to develop reliable data collection and data management procedures.

\section{Use of Coordinating Centers in Clinical Research}

When only one or even 2 sites are involved, and data collection and management requirements are not extensive, administrative and coordination often can be assumed by the organization with the leadership role for the study. However, when multiple sites are involved, coordination is both more critical and more difficult to accomplish by the entities with primary responsibility for site management.

Most large clinical trials, especially those involving several academic study sites, have independent centers that perform these "core" operational functions. NIH Cooperative Groups and Centers of Excellence often require that a separate entity support the primary research sites by assuming many of the administrative and data management tasks. These coordinating centers generally are required by the funder and are funded separately from the study sites. Budgets for coordinating centers typically range from $10 \%$ to $25 \%$ of the total budget of a project. However, most PBRNs are not involved in clinical trials, so the functions of coordinating centers in multi-PBRN studies would likely be less complex and, therefore, less expensive.
Use of Coordinating Centers in Primary Care Practicebased Research

Primary care research in general, and PBRN research in particular, suffers from a shortage of investigators. Time spent on administration and study coordination is time unavailable for member recruitment and for project development and conduct. Furthermore, many primary care departments have limited access to reliable data management and analytical support and insufficient experience managing multicenter projects. Thus, we propose that the services provided by coordinating centers could be equally useful and perhaps even more essential for multi-PBRN studies.

Although clinical researchers typically lack adequate funds to support and nurture collaborative research programs ${ }^{4}$ and use of separate coordinating centers to manage multi-PBRN research has so far been uncommon, the current trend in practicebased research calls for projects that require crossnetwork collaboration and sustainable infrastructure. ${ }^{5}$ With dedicated administrative and analytic support, the scope of projects that can be undertaken by regional PBRNs can expand, increasing the potential of coalitions of PBRNs to compete successfully for federal research funding.

The possible functions of a coordinating center in multi-PBRN studies are presented in Table 1. PBRN coordinating centers could be established ad hoc for each new multi-center or multi-network project. However, it probably would be more efficient and sustainable to establish centers that can be available to support multiple projects over time. In this model, the coordinating center would function as an integral component of the coalition of networks and provide supplemental services to secure additional funding and to support the momentum for the research agenda. For example, coordinating centers regularly monitor funding opportunities and could identify announcements of potential grants for practice-based research, evaluate the need for additional collaborators, and support the grant writing process. Resources could be applied across studies, standard procedures could be reused, and "lessons learned" could be identified and adopted.

\section{Examples of Multi-PBRN Studies Involving a Coordinating Center}

A regional PBRN (with which the first author is affiliated) had been conducting implementation re- 
Table 1. Potential Practice-based Research Network (PBRN) Coordinating Center Functions

\begin{tabular}{|c|c|}
\hline & Examples/Work Products \\
\hline \multicolumn{2}{|l|}{ Research functions } \\
\hline \multirow[t]{2}{*}{ Manage regulatory requirements } & - Adverse event management \\
\hline & - FDA reporting for clinical trials \\
\hline \multirow[t]{3}{*}{ Maintain standardization and quality control across sites } & - Common variable definitions \\
\hline & - Common coding structures \\
\hline & - Consistent data editing \\
\hline Monitor practice/provider/patient enrollment & - Produce study accrual reports \\
\hline \multirow[t]{4}{*}{ Develop and field data collection instruments } & - Surveys \\
\hline & - Interview guides \\
\hline & - Abstraction tools \\
\hline & - Implementation/process data forms \\
\hline Develop standards for procedures and data definitions & - Study-specific SOPs \\
\hline Create and operate a central data management system & - Produce analytic data files \\
\hline Perform quality control monitoring of adherence to protocol across all sites & - Produce monitoring reports \\
\hline Provide statistical expertise for design and development of research protocols & - Develop statistical analysis plans \\
\hline Provide statistical expertise for analysis and publication of study data & $\begin{array}{l}\text { - Draft analysis and findings sections for } \\
\text { scientific reports }\end{array}$ \\
\hline \multirow[t]{2}{*}{ Develop training materials and conduct training } & - In person \\
\hline & - Web-based/online \\
\hline Identify and contract with special experts or suppliers & - Subcontracts \\
\hline Develop and coordinate special panels or committees & - Minutes \\
\hline \multicolumn{2}{|l|}{ Administrative functions } \\
\hline \multirow[t]{2}{*}{ Coordinate project team communication } & - Set meeting agenda \\
\hline & - Schedule meetings \\
\hline \multirow[t]{3}{*}{ Manage study/project documentation } & - Track study milestones \\
\hline & - Manuals of procedures \\
\hline & - Decision documentation \\
\hline \multirow[t]{2}{*}{ Centralize storage and distribution of study materials } & - Materials inventory \\
\hline & - Version control \\
\hline \multirow[t]{2}{*}{ Prepare reports for governing and oversight bodies } & - Evaluation reports \\
\hline & - Quarterly reports \\
\hline \multirow[t]{2}{*}{ Support for dissemination } & - Prepare presentations/posters \\
\hline & - Manuscript preparation \\
\hline Assist with $\mathrm{OMB}$ packages & $\begin{array}{l}\text { - Assist PI with completion of application } \\
\text { materials }\end{array}$ \\
\hline
\end{tabular}

FDA, US Food and Drug Administration; OMB, Office of Management and Budgets; PI, primary investigator; SOP, standard operating procedure.

search since 1999, when infrastructure funding first became available from AHRQ. It therefore had the capacity to respond when the National Heart, Lung, and Blood Institute offered the opportunity to study implementation of evidence-based inter- ventions. The National Asthma Education and Prevention Program had recently produced updated guidelines for management of asthma, which were of interest to PBRN members, affiliated researchers, and clinical colleagues who had wanted 
to collaborate on an implementation project. Addressing the proposed research questions, however, required a cluster randomized trial with 4 study arms, and the principal investigator anticipated needing more practices than could be recruited from his PBRN alone. The decision was made to include a second regional network in a different state.

Having recently completed 2 multi-network projects, both of which presented administrative challenges for the primary investigator, and recognizing the need for additional methodological, analytic, and general management support, the investigators invited a large, private contract research organization (CRO) to serve as the coordinating center for the asthma guideline implementation study. The investigators and the CRO had been part of a National Institutes of Health (NIH) Roadmap project that included identifying best practices in both clinical and PBRNs. The CRO (with which the second two authors are affiliated), well known for data management and support of clinical trials, was interested in gaining experience coordinating research conducted in practice settings and led by PBRNs. The relationships established during the Roadmap project led the investigators and the research organization to develop the NIH proposal to conduct a cluster randomized trial of 4 implementation approaches to improve asthma care. The study includes 24 practices in each of 2 regional PBRNs, with the CRO providing the range of traditional coordinating center services (described below). Subsequently, the primary investigator and the CRO developed a larger AHRQ-sponsored study with 4 regional PBRNs to investigate methods to implement and diffuse evidence-based guidelines for management of chronic kidney disease (CKD).

For both studies, which are ongoing, the academic health centers/PBRNs are responsible for determining eligibility criteria for the study, practice recruitment, academic detailing, content of data collection instruments, hiring and supervision of practice facilitators, collection and transfer of survey data to the coordinating center, payment to practices and consultants, and analysis plans. The primary investigators and consultants also were responsible for responding to the clinician questions about the clinical guidelines.

The coordinating center assists with project administrative tasks including meeting regularly with the site directors and coordinators, drafting conference call agendas, hosting team meetings, recording decisions, and maintaining project documentation. It also hosts SharePoint web sites for each study to organize materials and input data (eg, practice facilitation visit report data) and monitors the study timeline to enable the project to stay on course. In addition, the coordinating center helps the primary investigators document study processes and decisions, develop recruitment materials, finalize data collection instruments, and prepare conference presentations and manuscripts. They are responsible for hiring and supervising medical record abstractors, collecting medical records abstraction data, managing all study data, developing preliminary analysis plans, and analyzing the process and qualitative and quantitative data.

Within the \$2.3 million budget for the asthma guideline implementation study, which involves 2 PBRNs, the coordinating center received approximately $\$ 740$ thousand (32\%). It received $28 \%$ of the $\$ 3$ million of the budget for the CKD guideline implementation study, which includes 4 PBRNs. Thus, the cost of the coordinating center was approximately the same as each PBRN for the asthma project and a little more than that for the CKD project.

\section{The AHRQ PBRN Resource Center}

To support primary care PBRNs throughout the United States, AHRQ established the PBRN Resource Center (RC) in 2002. Initially, the PBRN RC was created to provide support solely to PBRNs funded through AHRQ's PBRN initiative. In 2004, AHRQ expanded the mission to provide resources and assistance to all registered primary care PBRNs engaged in clinical and health services research. The PBRN RC staff and experts provide support to registered PBRNs through consultative services, informational resources, group learning experiences, and research tools. The PBRN RC also help plan and manage the annual AHRQ PBRN National Research Conference.

The AHRQ PBRN RC was asked to perform several specific tasks: (1) coordinate the annual PBRN meeting; (2) populate and maintain the PBRN database/registry; (3) maintain the public website and secure portal; and (4) provide consultation and support, especially for new and emerging networks, including organizing peer learning groups. Aside from developing some web-based 
data collection and management tools, providing coordinating center functions is beyond the scope of the PBRN RC. The only exception was one special contract under which the PBRN RC provided data management and analysis services for a multinetwork study of AHRQ's Safety in Office Practices instrument.

\section{Conclusions}

Regional PBRNs working together can increase the generalizability of practice-based research by increasing the number and diversity of the participating practices. Multinetwork studies capitalize on the close relationships and geographic proximity that exist with regional networks but are challenged to manage the additional coordination and operational requirements. Coordinating centers have been used successfully to improve the quality of multi-site clinical trials. However, multi-network PBRN studies have rarely included a coordinating center. We propose that PBRN researchers and funders begin to develop, fund, and use coordinating centers as a way to strengthen the PBRN re- search infrastructure and increase the reliability and generalizability of research results.

\section{References}

1. Green LA, Hickner J. A short history of primary care research networks: from concept to essential research laboratories. J Am Board Fam Med 2006;19: $1-10$.

2. Mold JW, Green LA. Primary care research: revisiting its definition and rationale. J Fam Pract 2000; 49:206-8.

3. Mold JW, Peterson KA. Practice-based research networks: working at the interface between research and quality improvement. Ann Fam Med 2005;3(Suppl 1):S12-20.

4. Coen SE, Bottorff JL, Johnson JL, Ratner PA. A relational conceptual framework for multidisciplinary health research centre infrastructure. Health Res Policy Syst 2010;8:29.

5. Pace WD, Fagnan LJ, West DR. The Agency for Healthcare Research and Quality (AHRQ) Practice-based research network (PBRN) relationship: delivering on an opportunity, challenges, and future directions. J Am Board Fam Med 2011;24: 489-92. 